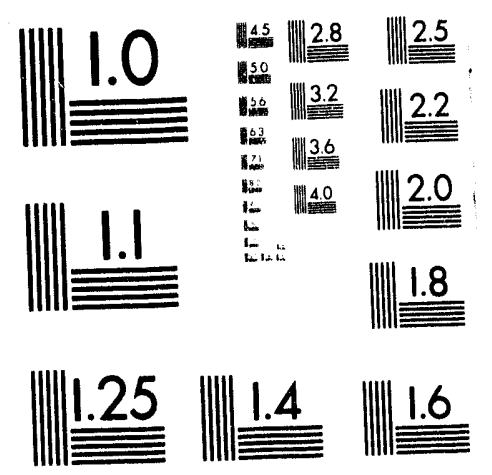



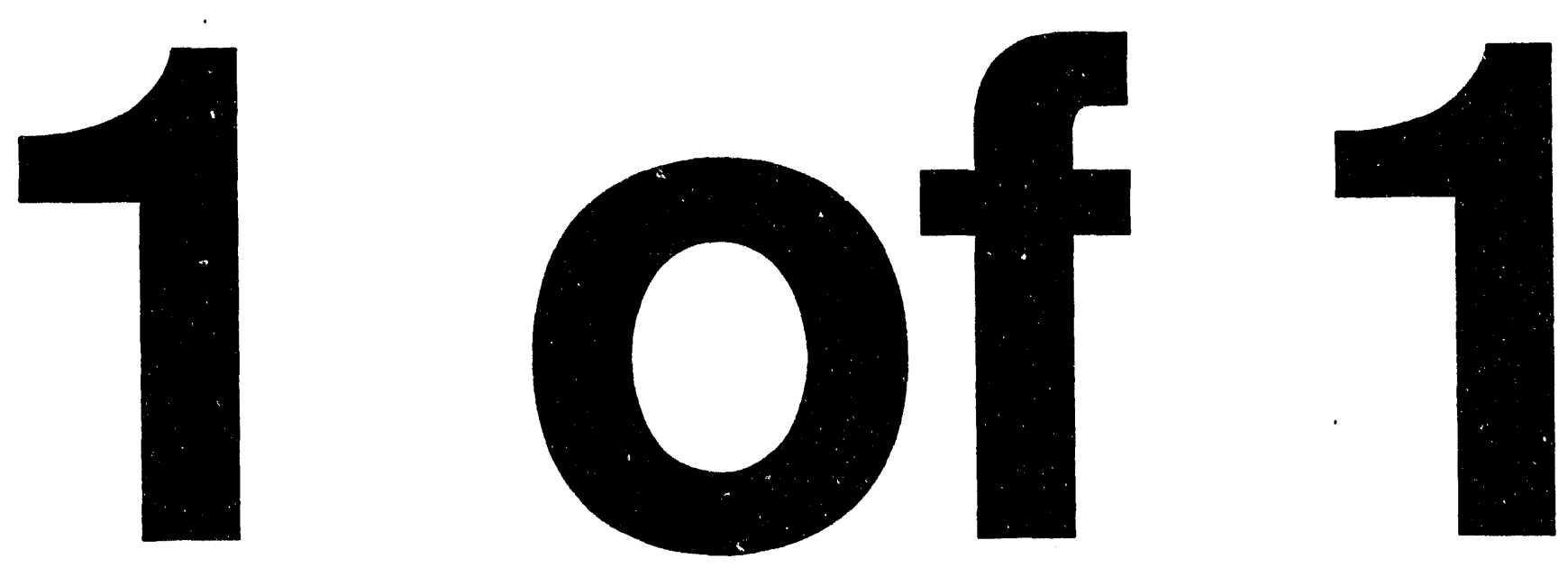


\title{
Alloy Sputtering at High Fluence: Preferential Sputtering and Competing Effects
}

\author{
Peter Sigmund ${ }^{a . c}$ and Antonino Oliva ${ }^{a . b}$ \\ a Physics Department, Odense Liniversity, \\ DK-5230 Odense M, Denmark . \\ ${ }^{b}$ Physics Department, University of Calabria, \\ I-87036 Rende, Italy \\ ${ }^{c}$ Physics Division, Argonne National Laboratory, \\ Argonne, IL 60439, USA
}

October 28, 1992

\begin{abstract}
This work addresses composition profiles and rartial sputter yields of an alloy under ion bombardment. The effects of preferential sputtering, mixing, and gibbsian segregation have been modelled. The theoretical basis is an integrodifferential equation derived previously, into which a feasible expression for a segregation current has been included. A dependence on composition of pertinent input parameters like relocation cross sections and atomic volumes has been allowed for but has not been explored explicitly.

In the limit of high fluence, a stationary state may be reached with a composition profile that shows more or less pronounced deviations from bulk stoichiometry. Two methods have been designed for direct determination of such stationary solutions. An iterative scheme for numerical solution of the nonlinear balance equation is very efficient and accurate. An approximate scheme, assuming weakly preferential behavior and thus based on a linearized balance equation, turns out to be quite accurate even in case of strongly preferential sputtering. An explicit analytical solution is given for a particular example.

On the basis of simple, schematic input, it is seen that the deviation of a stationary composition profile from bulk stoichiometry may be very strong, even when the sputter cross section is only weakly or moderately preferential. This difference is most pronounced in the absence of substantial feeding by mixing and segregation. In case of a nonzero depth of origin of sputtered atoms, composition profiles depend sensitively on the depth dependence of the sputter cross section, even in cases where consideration of only the partial sputter yields would suggest stoichiometric sputtering. A few examples illustrate explicitly the competition between preferential sputtering on the one hand, and mixing or segregation on the other.
\end{abstract}

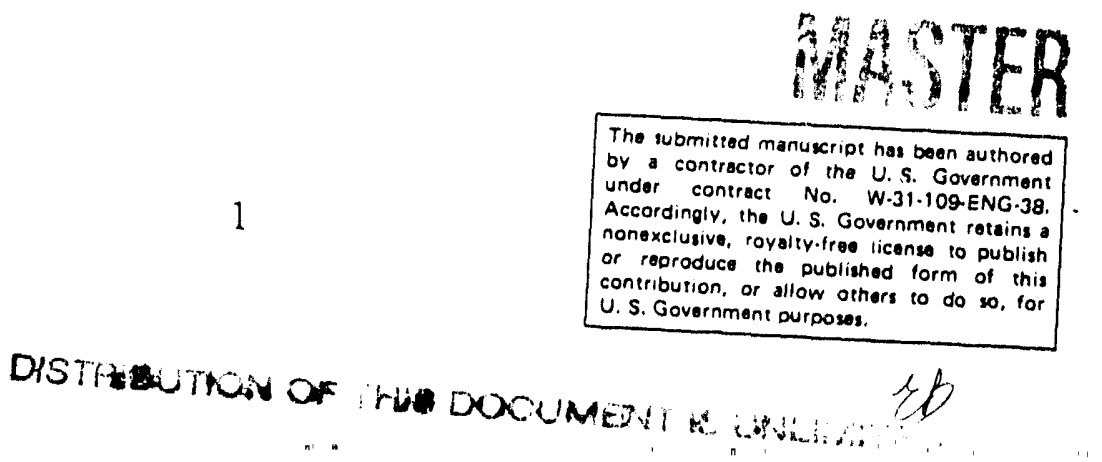




\section{Introduction}

Alloys and other multicomponent materials undergo compositional changes under ion bombardment [1]. The phenomenon has been know'n for many years [2], but its qualitative and quantitative understanding is making progress only slow.ly.

Until the mid 1970's, experimental evidence was very sketchy, and attempts at a theoretical understanding were directed mostly at modelling preferential sputtering [3]. Since altered layers were found to extend far beyond the depth of origin of sputtered atoms [4], compositional changes generated by preferential sputtering were assumed to . propagate into the bulk by diffusion [5].

Gradually it became clewi that ion bearns also cause mixing phenomena that are independent of what goes on at the surface $[6,7]$. Cascade mixing and recoil implantation are preferential [8]-[10] and need to be considered in the process of understanding compositional changes.

Increasing evidence showed that surface segregation cannot generally be ignored as a contributing factor [11]-[14].

The theory of alloy sputtering has been divided up into primary and secondary processes $[15,16]$, where primary processes are observable on a target with a given composition, exposed to a small ion fluence, while the complex of secondary effects stands for the accumulated composition changes after an arbitrary fluence. Another way of dividing up the field is atomistics and phenomenology, where atomistics deals with the pertinent cross sections for sputtering and mixing as well as other pertinent processes, and phenomenology is concerned with the general description of compositional changes, assuming that atomistic parameters are known. Much is still to be learned about either aspect.

The present work addresses the question of phenomenology. An attempt has been made to build up a consistent theory that allows some flexibility regarding the atomistic input. Such a description was outlined several years ago [17], but the theory turns out to be more general and powerful. The formalism to be developed will be fairly general. In view of a considerable number of competing effects, we find it desirable to study their contributions on the basis of simple models which might be able to quantify the instructive graphs that were introduced into the field by Andersen $[12.18,19]$.

In addition to sputtering, mixing, and segregation, there are two more ingredients. partly related to each other, that need to be incorporated into a theory of compositional changes. Firstly, collisional relocation of material generates density and pressure gradients that cannot persist in a stable material. Relaxation in response to pressure gradients must be allowed for. This is indispensible even when only sputtering is considered, since sputtering alone generates a near-surface layer depleted of atoms. The most common relaxation model assumes homogenous relaxation in planar geometry $[20,17]$. Secondly, the surface recedes because of the loss of material by sputtering. It is the receding surface that defines the natural coordinate system with respect to standard surface-analytical techniques. This motion of the coordinate system must be accounted for. 


\section{Recapitulation}

\subsection{Packing Condition for Target Configuration}

In planar geometry, a multicomponent target may be characterized by number densities $N_{i}(x)$, where $x$ is the depth from the surface, and $i=1,2, \ldots$ denotes the atomic species. In a stable target, the $N_{i}$ must obey some packing condition [17] within rather narrow limits,

$$
\sum_{i} \Omega_{i} N_{i}(x)=1,
$$

where $\Omega_{i}$ is the atomic volume of species $i$.

Two comments are appropriate here. Firstly, as it stands, $x$ is a continuous variable. A description of the target in terms of discrete layers of atoms is possible and has been carried out successfully [21]. The present notation readily allows for that specialization [22]. A discrete description of the structure of an alloy has certain advantages in case of a crystal, but it is also restricted in scope: No allowance has been made in such a model for the different atomic volumes of the species present, and no way has yet been pointed out how this important feature could be incorporated. Considering the $43 \%$ difference in number density between pure gold and pure copper, it seems questionable to represent a $\mathrm{CuAu}$ alloy by a constant number density, regardless of composition.

Secondly, the atomic volume $\Omega_{i}$ has usually been taken as a constant characterizing the species $i$, regardless of the other constituents $[17,20,23]$. One may argue that $\Omega_{i}$ might depend on the chemical environment and, hence, on the local composition. This option will be touched upon below.

\subsection{Balance Equation}

A balance equation describing the effects of sputtering, mixing, relaxation, and surface recession as a function of ion fluence $\Phi$ has been derived by us a decade ago [17],

$$
\frac{\partial N_{i}}{\partial \Phi}=L_{i} N_{i}-\frac{\partial}{\partial x}\left(N_{i} \int_{0}^{x} d x^{\prime} h\left(x^{\prime}\right)\right) \text {. }
$$

Here, $L_{i}$ is a relocation operator describing the primary process of mixing and sputtering according to

$$
L_{i} N_{i}(x)=\int_{0}^{\infty} d x^{\prime} N_{i}\left(x^{\prime}\right) G_{i}\left(x^{\prime}, x\right)-\int_{-\infty}^{\infty} d x^{\prime} N_{i}(x) G_{i}\left(x, x^{\prime}\right)
$$

where $G_{i}\left(x, x^{\prime}\right) d x^{\prime}$ is a relocation cross section, defined such that

$$
\delta \Phi N_{i}(x) d x G_{i}\left(x, x^{\prime}\right) d x^{\prime}
$$

is the mean number of $i$-atoms per unit area, relocated by a small ion fluence $\delta \Phi$ from a layer $(x, d x)$ to a layer $\left(x^{\prime}, d x^{\prime}\right)$. Moreover, $\mathrm{h}(\mathrm{x})$ is a relaxation function defined by

$$
h(x)=\sum_{j} \Omega_{j} L_{j} N_{j}(x) .
$$

Since eqn. (2) has been derived by two fairly different arguments [1T.23]. we shall refrain from going through either of these and instead discuss the physical significance of the individual terms. This will provide the ground for appropriate generalizations. 


\subsection{Sputtering and Mixing}

The term $L_{i} N_{i}(x)$ on the right-hand side of eq. (2) represents the primary effect of collisional mixing and sputtering before relaxation. Inspection of eq. (3) shows that there is a contribution due to sputtering.

$$
\left(L_{i} N_{i}\right)^{\text {sput }}=-N_{i}(x) \sigma_{i}(x)
$$

with $\sigma_{i}(x)=\int_{-\infty}^{0} d x^{\prime} G_{i}\left(x, x^{\prime}\right)$ representing relocation from inside $(x>0)$ to outside $\left(x^{\prime}<0\right)$ the surface. The term is negative since it implies a loss of material, and it is proportional to the number density of the respective species. The sputter cross section $\sigma_{i}(x)$ accounts for the relative depletion of species $i$ at depth $\mathrm{x}$. With this, the partial sputter yield $Y_{i}$ of the species $i$, i.e., the mean number of $i$-atoms sputtered per incident primary particle reads [17]

$$
Y_{i}=\int_{0}^{\infty} d x N_{i}(x) \sigma_{i}(x) .
$$

The remainder of eq. (3),

$$
\left(L_{i} N_{i}\right)^{m i x}=\int_{0}^{\infty} d x^{\prime}\left[N_{i}\left(x^{\prime}\right) G_{i}\left(x^{\prime}, x\right)-N_{i}(x) G_{i}\left(x, x^{\prime}\right)\right],
$$

accounts for transport of material within the target $\left(x, x^{\prime}>0\right)$, i.e., for the primary effect of collisional mixing.

The second term on the right-hand side of eq. (2) accounts for relaxation and surface recession. Indeed, multiplication of eq. (2) by $\Omega_{i}$, assuming $\Omega_{i}$ independent of the composition, and summation over all $i$ shows that the stability condition eq. (1) is fulfilled everywhere in the halfspace $x>0$, provided that $h(x)$ is defined according to eq. (4).

Eq. (2) may be cast into the form

$$
\frac{\partial N_{i}}{\partial t}+\frac{\partial J_{i}^{\text {relax }}}{\partial x}=J_{0} L_{i} N_{i}
$$

where $J_{0}(t)=d \Phi / d t$ is the current density of incoming ions, and

$$
J_{i}^{\text {relax }}=J_{0} N_{i} \int_{0}^{x} d x^{\prime} h\left(x^{\prime}\right)
$$

a relaxation current that accounts for the combined effect of relaxation and surface recession.

Note that the relaxation current vanishes at the surface: Indeed, the loss of matter by sputtering is fully accounted for by eq. (5). The function of the relaxation term with regard to sputtering is to adjust the density within the target to the packing condition and to redefine the position of the surface.

Also the primary relocation term in eq. ( $T$ ) can be expressed by a current density,

$$
J_{i}^{\text {prim }}=J_{0} \int_{x}^{\infty} d x^{\prime} L_{i} N_{i}\left(x^{\prime}\right)
$$

Here, the integration constant was chosen such as to ensure that $\left.J_{i}^{\text {prim }}\right|_{x=0}=-J_{0} Y_{i}$, i.e., the sputter current.

For the primary mixing current, we have

$$
J_{i}^{\text {prim,mix }}=J_{0} \int_{x}^{\infty} d x^{\prime}\left[L_{i} N_{i}\left(x^{\prime}\right)\right]^{m i x}=-J_{0} \int_{0}^{x} d x^{\prime}\left[L_{i} N_{i}\left(x^{\prime}\right)\right]^{m i x},
$$

since this current vanishes at the surface. 


\subsection{Input Parameters Depending on Local Composition}

Collision cross sections depend on the atomic numbers and masses of the colliding atoms. For a multicomponent material, this implies that penetration parameters depend on the composition. Therefore, the relocation operator $L_{i}$ will in general be a functional of the number densities $N_{j}(x)$ of all constituents of the medium, and that functional dependence will sample a range of $x$-values of the order of the penetration. depth of the beam. Relocation cross sections utilized in analytical as well as numerical work on ion beam mixing have frequently been based on the average composition of the stopping medium. While it may be a nontrivial task to account for local variations in composition explicitly, that feature is readily contained in the general equations. Therefore, the expression $L_{i} N_{i}(x)$ is to be understood as the primary change in $N_{i}$ at depth $x,\left(\partial N_{i} / \partial \Phi\right)^{\text {prim }}$, whatever its dependence on $N_{i}$ and the other densities. Thus, $L_{i}$ will not in general be a linear operator.

As mentioned above, also the atomic volume may depend on the local composition, $\Omega_{i}=\Omega_{i}\left\{N_{1}(x), N_{2}(x), \ldots\right\}$, but unlike the variation of the relocation operator, this dependence may be taken to be local and governed by the chemical environment.

This feature can be incorporated into the balance equation by a generalization of the procedure outlined in ref. [17]. Let eq. (1) be fulfilled initially; a fluence increment $\delta \Phi$ causes a primary change in density $\delta N_{i}$ which, in turn, causes a change in atomic volume, $\delta \Omega_{i}=\sum_{j}\left(\partial \Omega_{i} / \partial N_{j}\right) \delta N_{j}$. This leads to a deviation from the paching condition eq. (1),

$$
\sum_{i} \Omega_{i} N_{i} \rightarrow \sum_{i}\left(\Omega_{i}+\delta \Omega_{i}\right)\left(N_{i}+\delta N_{i}\right)=1+\epsilon
$$

with

$$
\epsilon=\sum_{i}\left(\Omega_{i}+\sum_{j} \frac{\partial \Omega_{j}}{\partial N_{i}} N_{j}\right) \delta N_{i},
$$

up to first order in $\delta N_{i}$. This serves as the starting point of a relaxation calculation which may proceed as in ref. [17], with the result that the function $h(x)$ in e?. (4) needs to be amplified to

$$
h(x)=\sum_{j}\left(\Omega_{j}+\sum_{k} \frac{\partial \Omega_{k}}{\partial N_{j}} N_{k}\right) L_{j} N_{j}
$$

For this derivation to make sense, it is necessary to assume that the atomic volume $\Omega_{i}$ at any given depth does not change under homogeneous compression or dilatation. This assumption, which is an immediate consequence of the physical requirement that the atomic volume is completely determined by the chemical environment, implies that

$$
\sum_{j} N_{j} \frac{\partial \Omega_{i}}{\partial N_{j}}=0
$$

In the appendix, a proof is given that the stability condition eq. (1) is satisfied at all fluences if $N_{i}$ obeys eq. (2) with $h(x)$ given by eq. (10). That proof makes explicit use of eq. (11). 


\subsection{Surface Segregation}

An attempt will now be made to incorporate surface segregation into the theoretical scheme. The pertinent physical processes have been described in numerous sources [24]-[26]. The present picture is a modification of the one formulated in ref. [21].

Surface (or Gibbsian) segregation is driven by a difference in surface energy of the various constituents, and it is activated either by elevated temperature or, at ambient temperature, through the energy deposited in the target by a bombarding ion.

In a discrete-layer model, the elementary event in surface segregation is the interchange of positions between two atoms in the top layer and the nearest subsurface layer. In principle, also deeper layers may participate in the process. In more general terms, this is equivalent with a set of segregation currents, one for each species, plus an inverse collective current that ensures the packing condition to be fulfilled. The net result will be that the species with the dominating segregation current will be enriched most strongly at the surface, while all others will be enriched less strongly or depleted.

Consider first a dilute solution of some component $i$ in a matrix, and assume that $i$-atoms tend to segregate at the surface. In equilibrium, one expects a concentration profile of the type of

$$
N_{i}(x)=N_{i}(\infty) \exp \left(\frac{-V_{i}(x)}{k T}\right),
$$

where $V_{i}(x)$ is a potential energy which falls below its bulk value $V_{i}(\infty)=0$ in a shallow region near the surface.

An ansatz for a segregation current consistent with the above looks as follows,

$$
J_{i}^{s e g}=-D_{i} \frac{\partial}{\partial x}\left[N_{i}(x) \exp \left(\frac{V_{i}(x)}{k T}\right)\right],
$$

where $D_{i}$ is a pertinent diffusion coefficient. Indeed, the current vanishes when $N_{i}(x)$ has reached the stationary state (12). If $N_{i}$ is constant, $J_{i}^{\text {seg }}$ will be directed in the negative $x$-direction. The magnitude of the current is determined both by $V_{i}$ and by $D_{i}$. A dependence on time may be implicit in the latter.

Consider now a medium in which each component has been assigned a segregation current of the form of eq. (13). It is now reasonable to assume that the potential function $V_{i}(x)$ may be either positive or negative, but it should always be vanishing inside the bulk. The total volume current through a plane at $x$ will be given by $J^{\text {vol }}=$ $\sum_{i} \Omega_{i} J_{i}^{\text {seg }}$. This current needs to be compensated by a stoichiometric (or "collective") current $[27,23] J_{i}^{\text {coll }}=-N_{i} J^{\text {vol }}$, such that the total volume current vanishes, i. e.,

$$
\sum_{i} \Omega_{i}\left(J_{i}^{\text {seg }}-N_{i} J^{\text {vol }}\right)=0 .
$$

This relation is easily seen to be fulfilled by means of the packing condition (1).

From the equation of continuity, one finds

$$
\frac{\partial N_{i}}{\partial t}+\frac{\partial}{\partial x}\left(J_{i}^{\text {seg }}-N_{i} \sum_{j} \Omega_{j} J_{j}^{\text {seg }}\right)=0 .
$$

This is the kinetic equation describing surface segregation, written in sufficiently general terms to allow for incorporation of sputtering and mixing. 
The topic of surface segregation in connection with sputtering is delicate. It involves the question of boundary conditions as well as stability in the absence of irradiation. The segregation potential $V_{i}(x)$ may also be related physically with the compositionindependent atomic volume mentioned above. Not all those problems can be attacked in the present paper.

\subsection{Balance Equation Including Sputtering, Mixing, and Se- gregation}

The combined effect of sputtering, mixing, and segregation may be written in the form

$$
\frac{\partial N_{i}}{\partial t}+\frac{\partial}{\partial x}\left(J_{i}^{\text {seg }}+J_{i}^{\text {prim }}\right)-\frac{\partial}{\partial x} N_{i} \sum_{j} \Omega_{j}\left(J_{j}^{\text {seg }}+J_{j}^{\text {prim }}+J_{0} Y_{j}\right)=0,
$$

where $J_{i}^{\text {prim }}$ is given by eq. (9) and $Y_{i}$ by eq. (6). It is seen that eq. (15) reduces to (14) in the absence of mixing and sputtering, and to eq. (2) in the absence of segregation. Here, any composition dependence of the atomic volumes $\Omega_{i}$ has been ignored.

By introduction of a total primary current $J_{i}$,

$$
\begin{aligned}
J_{i}(x) & =J_{i}^{\text {seg }}+J_{i}^{\text {prim }} \\
& =-D_{i} \frac{\partial}{\partial x}\left[N_{i}(x) \exp \left(\frac{V_{i}(x)}{k T}\right)\right]+J_{0} \int_{x}^{\infty} d x^{\prime} L_{i} N_{i}\left(x^{\prime}\right),
\end{aligned}
$$

this reduces to

$$
\frac{\partial N_{i}}{\partial t}+\frac{\partial}{\partial x}\left(J_{i}-N_{i} \sum_{j} \Omega_{j} J_{j}\right)=v \frac{\partial N_{i}}{\partial x}
$$

where

$$
v=v(t)=J_{0} \sum_{j} \Omega_{j} Y_{j}
$$

is the recession speed of the surface. According to eq. (16), the boundary conditions on the primary current read

$$
\begin{aligned}
J_{i}(0) & =-J_{0} Y_{i} \\
J_{i}(\infty) & =0 .
\end{aligned}
$$

\section{Solving the Balance Equation}

\subsection{General Considerations}

In the following we consider solutions of eq. (17). This implies that we stick to the assumption of a constant atomic volume, but otherwise, both sputtering, mixing, and segregation will be allowed for. Solutions in the limit of small fluence may easily be found. More interesting in practice are solutions for the stationary state that may be reached at high fluence. Apart from two exceptions $[17,28]$, such solutions have so far been determined numerically via the time-dependent equation [29].

A necessary condition for a stationary state to be reached is that the target initially be homogeneous, i.e.,

$$
N_{i}(x)_{t=0}=c_{i}=\text { const. }
$$


for $x \geq 0$. This assumption will be applied throughout the remainder of the paper. Here, the $c_{i}$ have to obey the packing condition (1),

$$
\sum_{j} \Omega_{j} c_{j}=1
$$

\subsection{Low-Fluence Limit}

In the low-fluence limit $(t=0)$, we may set $N_{i}=c_{i}$ in eq. (17) to obtain

$$
\left(\frac{\partial N_{i}}{\partial t}\right)_{t=0}=c_{i} \sum_{j} \Omega_{j} c_{j}\left(I_{j}-I_{i}\right)
$$

where

$$
I_{i}=-\frac{d}{d x} D_{i} \frac{d}{d x} \exp \left(\frac{V_{i}(x)}{k T}\right)-J_{0} L_{i}
$$

and

$$
L_{i}=\int_{0}^{\infty} d x^{\prime} G_{i}\left(x^{\prime}, x\right)-\int_{-\infty}^{\infty} d x^{\prime} G_{i}\left(x, x^{\prime}\right) .
$$

Thus, for a small time increment $\delta t$,

$$
N_{i} \simeq c_{i}\left[1+\delta t \sum_{j} \Omega_{j} c_{j}\left(I_{j}-I_{i}\right)\right] .
$$

It is seen that the species $i$ with the largest, current $I_{i}$ is depleted. In the particular case of a binary target, eq. (23) reduces to

$$
N_{1} \simeq c_{1}\left[1+\delta t \Omega_{2} c_{2}\left(I_{2}-I_{1}\right)\right]
$$

and vice versa for $N_{2}$.

\subsection{High-Fluence Limit: Iterative Solution}

In the stationary state $(t=\infty)$, the time derivative in eq. (17) drops out. Integration from 0 to $x$ under observation of the boundary condition (19) yields

$$
J_{i}-N_{i} \sum_{j} \Omega_{j} J_{j}+J_{0} Y_{i}=v N_{i}
$$

Conversely, integration from $x$ to $\infty$ under observation of the boundary condition (20) yields

$$
-J_{i}+N_{i} \sum_{j} \Omega_{j} J_{j}=v c_{i}-v N_{i},
$$

since $N_{i}(x)_{x=\infty}=c_{i}$ if a stationary state exists. Adding the two equations yields

$$
Y_{i}=c_{i} \frac{v}{J_{0}}=c_{i} \sum_{j} \Omega_{j} Y_{j},
$$

by means of eq. (18). It is seen that the partial sputter yield $Y_{i}$ of species $i$ is proportional to the bulk concentration $c_{i}$ in the stationary state. This is a central property of 
the partial sputter yield [1]. It emerges here as a result of the general formalism. yet under the assumption that a stationary state exists. Note that this result is unaffected by the detailed nature of the mixing and segregation currents.

By means of eq. (1), eq. (24) may be uritten in the form

$$
v\left(N_{i}-c_{i}\right)=\sum_{j} \Omega_{j}\left(N_{j} J_{i}-N_{i} J_{j}\right)
$$

or

$$
N_{i}=c_{i}+\frac{1}{v} \sum_{j} \Omega_{j}\left(N_{j} J_{i}-N_{i} J_{j}\right)
$$

This relation can be utilized as a scheme for iterative solution of the balance equation in the stationary state. This involves eqs. $(6,16,18)$, which express $J_{i}$ and $v$ by $N_{i}$.

An alternative iteration scheme can be found by adding and subtracting a term $c_{i} \sum_{j} \Omega_{j} J_{j}$ in eq. (24), so that

$$
N_{i}=c_{i}+\frac{\sum_{j} \Omega_{j}\left(c_{j} J_{i}-c_{i} J_{j}\right)}{v+\sum_{j} \Omega_{j} J_{j}}
$$

This scheme may be particularly useful in situations where the recession speed $v$ is small.

\subsection{High-Fluence Limit: Weak Preferentiality}

If neither sputtering, mixing, nor segregation are strongly preferential, the balance equation may be linearized in terms of the deviation from stoichiometry [17]. We set $J_{i}=S_{i} N_{i}$, with $S_{i}$ being an operator defined by eq. (16), and $N_{i}=c_{i}+\Delta N_{i}$, $S_{i}=S+\Delta S_{j}$ with $S=\sum_{j} \Omega_{j} c_{j} S_{j}$. After linearization in terms of the small parameters $\Delta S_{i}, \Delta N_{i}$, we obtain from eq. (25)

$$
S \Delta N_{i}-\Delta N_{i} S-v \Delta N_{i}=-c_{i} \Delta S_{i}
$$

For simplicity, $S_{i}$ has been taken to be a linear operator here. Eq. (28) is-a linear integro-differential equation that determines $\Delta N_{i}$. An explicit solution will be given below.

\section{Examples}

In the following, we shall consider a target with a homogeneous initial composition, cf. eq. (21), and the $c_{i}$ satisfying the packing condition eq. (22). The low-fluence limit will be mentioned but attention will be paid mostly to the stationary state at high fluence. Numerical examples refer to binary alloys.

\subsection{Preferential Sputtering}

When mixing and segregation are ignored, the relocation operator $L_{i} N_{i}$ reduces to $-\sigma_{i}(x) N_{i}(x)$ according to eq. (5). The limit of low fluence, eq. (23) reads

$$
N_{i} \simeq c_{i}\left[1+\delta \Phi \sum_{j} \Omega_{j} c_{j}\left(\sigma_{j}-\sigma_{i}\right)\right] \text {, }
$$


where $\delta \Phi=J_{0} \Delta t$ is the fluence increment. This expresses the expected result that the component with the largest sputter cross section suffers the most pronounced depletion on a relative scale. Conversely; the component with the smallest sputter cross section gets enriched. These statements hold both for the partial yields as well as their dependence on depth.

For a binary target, eq. (29) reduces to

$$
N_{1} \simeq c_{1}\left[1+\delta \Phi \Omega_{2} c_{2}\left(\sigma_{2}-\sigma_{1}\right)\right] .
$$

Next, consider the stationary state. With the functions

$$
f_{i}(x)=\int_{0}^{x} d x^{\prime} \sigma_{i}\left(x^{\prime}\right) N_{i}\left(x^{\prime}\right)
$$

and

$$
g_{i}(x)=\int_{x}^{\infty} d x^{\prime} \sigma_{i}\left(x^{\prime}\right) N_{i}\left(x^{\prime}\right),
$$

eq. (26) reads

$$
N_{i}=c_{i}+\frac{\sum_{j} \Omega_{j}\left(N_{i} g_{j}-N_{j} g_{i}\right)}{\sum_{j} \Omega_{j} Y_{j}} .
$$

Similarly, eq. (27) reads

$$
N_{i}=c_{i}+\frac{\sum_{j} \Omega_{j}\left(c_{i} g_{j}-c_{j} g_{i}\right)}{\sum_{j} \Omega_{j} f_{j}} .
$$

These relations are valid only provided that a stationary state exists. As an obvious counterexample where such is not the case, assume that only one component, say $i=1$, has a nonvanishing sputter yield. Then, uncritical application of eq. (31) or eq. (32) would lead to unphysical results.

Finally, the case of weak preferentiality, as determined by eq. (28) leads to

$$
N_{i}(x) \simeq c_{i}\left(1-\sum_{j} \Omega_{j} c_{j} \int_{x}^{\infty} d x^{\prime} \frac{\sigma_{i}\left(x^{\prime}\right)-\sigma_{j}\left(x^{\prime}\right)}{\sum_{j} \Omega_{j} c_{j} \int_{0}^{x^{\prime}} d x^{\prime \prime} \sigma_{j}\left(x^{\prime \prime}\right)}\right) .
$$

This expression conceals a logarithmic singularity near $x=0$. While the very appearance of a singularity must be an artefact of the series expansion in terms of weak preferentiality, it also indicates that major composition changes accumulate at the surface even in case of weakly preferential sputtering. This important observation, which will be exemplified below, is related to the nonlinearity of the balance equation.

Explicitly, for a binary medium, we find

$$
N_{1}(x) \simeq c_{1}\left(1+c_{2} \Omega_{2} \int_{x}^{\infty} d x^{\prime} \frac{\sigma_{2}\left(x^{\prime}\right)-\sigma_{1}\left(x^{\prime}\right)}{\sum_{j} \Omega_{j} c_{j} \int_{0}^{x^{\prime}} d x^{\prime \prime} \sigma_{j}\left(x^{\prime \prime}\right)}\right)
$$

and vice versa for $N_{2}(x)$.

\subsection{Exponential Sputter Cross Section}

For the purpose of getting qualitative insight, we apply a a simple, schematic. yet quite realistic [22] sputter cross section [17][30],

$$
\sigma_{i}(x)=A_{i} \exp \left(-x / a_{i}\right)
$$


where the constants $a_{1}$ represent the depths of origin of the species present $w$ hile the amplitude factors $A_{i}$ determine the surface depletion. The initial sputter yields for the homogeneous target reduce to $Y_{i}=c_{i} A_{i} a_{i}$, according to eq. (6).

Stationary solutions have been found by iteration on the basis of eq. (31) or (32). Accurate results have been produced by both procedures, but in case of strongly preferential sputtering. instabilities were found in the procedure based on eq. (32). Therefore, all examples mentioned here have been determined from eq. (31). Typically, one iteration takes about a second on' a personal computer. 10-20 iterations are sufficient to generate approximate profiles if a homogenous target is chosen as the starting point for the iteration. Accurate solutions, leading to stoichiometric partial sputter yields within a relative accuracy of $10^{-5}$, require typically $100-200$ iterations.

A particularly simple analytical solution is found when the difference in depth of emergence between the species is ignored, i.e., $a_{1}=a_{2}=a$. For a binary mediurn, eq. (30) predicts

$$
N_{1}(x) \simeq c_{1}\left(1+\delta \Phi c_{2} \Omega_{2}\left(A_{2}-A_{1}\right) e^{-x / a}\right)
$$

at low fluence. At high fluence, for weak preferentiality, eq. (33) yields

$$
N_{1}(x) \simeq c_{1}\left(1+c_{2} \Omega_{2}\left(A_{2}-A_{1}\right) \frac{-\log \left(1-e^{-x / a}\right)}{\Omega_{1} c_{1} A_{1}+\Omega_{2} c_{2} A_{2}}\right)
$$

for the stationary state. Figure 1 shows a comparison between the exact stationary profile and eq. (35). With the chosen sputter parameters, the initial sputter yields differ by a factor of 2 . Despite this pronounced nonpreferential behavior, the approximation of weak preferentiality generates a profile that agrees surprisingly well with the exact solution.

In figure 1, as in all subsequent figures, no units have been assigned to the abscissa scale. In figure 1 , the quantity $a(=1)$ denotes the mean depth of origin of sputtered (1 or 2) atoms. Subsequent graphs should be read correspondingly, apart from the fact that the two depths may differ.

The behavior of the two m-in curves shown in figure 1 is dramatic in the immediate vicinity of the surface. The analytical approximation turns negative: this unphysical behavior is related to the the logarithmic singularity at $x=0$. Yet the point where this happens is so close to the surface that it is of little practical importance. Next, the exact concentration profile of the preferentially sputtered component is found to go to zero at $x=0$. It will be demonstrated below that this is not an artefact of the numerical procedure. The feature occurs when the ratio $A_{1} / A_{2}$ between the amplitudes of the sputter cross sections exceeds a certain critical value. The main point to be noted is that the variation with depth of the stationary profile is substantially steeper than the variation in the underlying sputter cross section in which it originates. In order to illustrate this point. a low-fluence profile, eq. (34), scaled to the high-fluence profile at large depth, has also been included in figure 1.

Figure 2 shows a comparison of exact stationary profiles for different depths of origin but identical amplitudes of the sputter cross section. The amplitude ratio $A_{1} / A_{2}$ is 1.5 in all cases. The ratio of partial sputter yields $Y_{1} / Y_{2}$ varies between 1 and 2.25 . Figure 3 shows a similar comparison. now with the depths of origin kept constant at $a_{1} / a_{2}=1.5$ but varying amplitude. The ratio of partial sputter yields goes through the same sequence as in figure 2. These graphs illustrate the distinct difference between the effect of the amplitude and that of the depth of origin on the composition profile. 
The lowest curves $(c)$ in both figures (these curves are identical) illustrate the case where species 1 is sputtered preferentially due to larger amplitude and greater depth. At an initial sputter yield ratio of 2.25 , the preferentially sputtered species is depleted to a surface concentration of $4 \%$.

In the intermediate case (b), species 1 is sputtered preferentially either because of greater amplitude only (figure 2) or greater depth only (fig. 3). Not surprisingly, the depletion in the former case is most pronounced near the surface. The magnitude of the difference is more striking: at a (rather moderate) initial yield ratio of 1.5 , the concentration of species 1 at the very surface goes down from $50 \%$ at zero fluence to $10 \%$ in the stationary state. In the latter case, the corresponding level is $35 \%$. The initial sputter yield ratios are identical.

Another interesting feature shows up in the uppermost curves (a) which are mirror images of each other. Parameters have been chosen so that the partial sputter yields are identical ia the low-fluence limit, $Y_{1}=Y_{2}=n .75$ in the chosen units. Nevertheless, sputtering is not stoichiometric because differenc depth ranges are depleted at different rates in the two cases. As a result, the stationary profiles differ substantially up to about half the average sputter depth. The species with the larger amplitude shows a surface concentration of only $17 \%$, while the species with the larger sputter depth is correspondingly enriched to $83 \%$. In the absence of competing effects like mixing or surface segregation, a low-energy ion scattering measurement would suggest species 1 to be sputtered preferentially at a yield ratio $0.83 / 0.17 \simeq 5$, despite the fact that the homogeneous target sputters stoichiometrically. At the same time, the partial sputter yields have only decreased slightly from their initial value 0.75 to their stationary values $Y_{1}=Y_{2}=0.72680$ in the chosen units.

Figures 4 and 5 show similar results as figures 2 and 3 with a modified choice of parameters where the amplitude ratio varies more strongly. It is seen that for an amplitude ratio of 2 , complete depletion happens at the surface in the preferentially sputtered component for the range of depth parameters chosen.

Conversely, figure 6 shows results where the depth parameters differ by a factor of two but the amplitude ratio varies less pronouncedly. Even in the lowermost curve (c), where both amplitude and depth dependence favor preferential sputtering of component 1 , that species is not depleted completely at the surface. This was made sure by an exceptionally large number of iterations. We note that here, as well as in the lowermost curve of figs. 4 and 5 (which are identical), the initial yield ratio is 3.0 . This is to be compared with a value of 2.0 in figure 1, where complete depletion was observed. Clearly, the amplitude factor is more influential than the depth parameter with regard to the stationary concentration at the very surface.

The present calculations say little about the bombardment fluence that is necessary to arrive at a stationary profile. We may compare eq. (34) with eq. (35) at large depths to obtain the effective bombardment fluence $\Phi^{\text {sat }}$ to achieve saturation at large depths,

$$
\Phi^{\text {sat }}=\frac{1}{\Omega_{1} c_{1} \cdot A_{1}+\Omega_{2} c_{2} \cdot A_{2}}=\frac{a}{d x / d \Phi}
$$

where $d x / d \Phi=v / J_{0}$ is the surface recession rate per fluence. While this is a reasonable result for large depths, one should expect that the pronounced surface depletion effects that emerge from figures 1-6 require higher bombardment fluences. 


\subsection{Mixing, Segregation, and Diffusion}

The main purpose of the present section is not to let the results of the previous section stand alone. A discussion of the competition between preferential sputtering on the one hand and mixing, segregation and diffusion on the other at the level of detail of the previous section is a major computational program which has been started but not completed yet. A program of this type was carried out several years ago on a model system in which a great number of effects were included, but the sputter process itself as well as collisional mixing were described more schematically, in a complimentary model disregarding depth effects and volume relaxation (for a review cf. [21]).

Figure 7 shows the stationary profile including mixing, sputtering, and relaxation, evaluated for an exponential relocation cross section [17]

$$
G\left(x, x^{\prime}\right)=B_{i} \exp \left(-b_{i}\left|x-x^{\prime}\right|\right)
$$

This relocation cross section models isotropic cascade mixing, and the relative significance of cascade mixing and sputtering is not unrealistic with regard to the order of magnitude. We note reasonable but by no means perfect agreement with results found many years ago by numerical solution of the time-dependent balance equation $[29,28]$. In those results, the yield ratio exceeded the stoichiometric ratio by $\simeq 7 \%$. We have also included the original result which was hinging on weak preferentiality as well as a smoothness assumption [17]. The latter result is known to be inaccurate. The point to note is the fact that when mixing is added to sputtering, the preferentially sputtered component is no longer depleted completely at the surface. Clearly, cascade mixing feeds 1-atoms into the surface region, and thus prevents excessive composition gradients from developing.

This point is illustrated more explicitly in fig. 8 . Here, the sputter current has been separated from the mixing current. The sputter current was left unchanged while the mixing current was scaled by a factor $f$. It is seen that with increasing mixing current, the steep concentration gradient generated by preferential sputtering is weakened. Note that in this particular example. both sputtering and mixing are preferential. As it turns out, increasing the factor $f$ beyond $f=2$ does not produce a noticeable change. Hence, the profile for $f=2$ is very close to being caused by preferential mixing, without a substantial contribution from preferential sputtering.

Figures 9-11 show profiles generated by simultaneous sputtering and segregation. In the computations, boundary conditions were imposed, and we had greater difficulties in arriving at stable solutions. These graphs are less accurate in general and based on a smaller number of iterations. They have been included here to show a few trends, but they should be taken with caution.

In figure 9, species 1 is sputtered preferentially while species 2 segregates at the surface. Hence, both effects act in the direction of species 1 being depleted near the surface. The two curves refer to preferential sputtering by different amplitudes and depth, respectively. Diffusion coefficients were chosen quite small. Nevertheless, the effect of segregation is clearly distinguished in the near-surface region since quite pronounced segregation potentials were chosen, $V(0) / k T= \pm 2$.

Figures 10 and 11 show sets of profiles representing the opposite situation where the preferentially sputtered component segregates. With the range of ion currents chosen, preferential sputtering dominates over most of the depth range, resulting in depletion of 
species 1, while segregation acts in the opposite direction in the vicinity of the surface. This effect is current-dependent: The smaller the ion current, the more pronounced is the contribution due to segregation. Figure 10 represents depth-preferential sputtering and figure 11 amplitude-preferential sputtering. As was to be expected, the latter case shows more pronounced variations in the vicinity of the surface.

\section{Discussion and Summary}

We like to briefly recapitulate what has been achieved here and what remains to be done.

While the balance equation as a basis for the theory of sputtering of multicomponent targets has been established a decade ago, it has up till now been useful mainly as a tool in calculations on ion beam mixing [31]. One reason for its rather minor impact on sputter theory was the apparent lack of ability to accomodate surface segregation. With a segregation current as proposed here, that effect should be fully incorporated in the phenomenological description.

While the primary relocation term in the balance equation has been written in a way that is suggestive of the relocation operator being linear, such a linearity is not implied in the definition. Hence there are no inherent limitations on the level of accuracy of realistic atomistic input regarding mixing and preferential sputtering. The description is applicable equally well to single and polycrystals, and full allowance may be made for the influence of the varying composition profile on relocation cross sections as well as atomic volume.

On the other hand, the description is one-dimensional as it stands, with an average taken over the lateral dimension. This feature is also common to most existing computer codes simulating high-fluence sputtering behavior [32]. The limitations imposed by this feature need to be looked into.

The notation is suggestive of a continuum description of the composition profile, and indeed, at least the relaxation model utilized here appears difficult to reconcile with a discrete description based on a fixed lattice structure. In that sense, the present description is complementary to the one developed by Lam and coworkers [21], where relaxation is allowed for by means of currents of migrating point defects. Point defects are not part of a description that is based on homogeneous relaxation following a unique stability criterion. In that respect the lattice model is superior. On the other hand, both mixing and sputtering are dealt with as diffusional processes in a lattice model, while transport of material over a substantial depth interval, and pressure changes resulting thereof, are disregarded. As mentioned in the introduction, changes in lattice constant brought about by substantial changes in local composition have not been accomodated in the lattice model although they possibly could be.

The explicit solutions given in the present paper are based on schematic input. No attempt has been made to identify explicit results as being representative for a specific system. While we have made an effort to provide accurate numerical solutions to the balance equation, this effort was indicated by the desire to understand properties of the balance equation and its solutions. The question of how to interpret these solutions in relation to processes happening within a very shallow depth scale in a realistic medium needs to be answered, but the present paper gives only qualitative hints in 
that direction.

The combined effect of sputtering and relaxation has been studied in some detail. Numerical solutions of the balance equation have been found to be stable in that particular case. We find it striking that a factor of two difference in sput.ter amplitude between two components in a binary alloy is sufficient to get the surface concentration of the preferentially sputtered component down to zero (figure 1). Even more striking are the differences found in composition profiles expressed by curves labelled $a$ in figures 2 and 3: These curves demonstrate that preferential sputtering is not just a matter of different partial sputter yields, but that the depth dependence of the sputter cross section may be crucial. In the case in question, partial sputter yjelds are identical in the low-fluence limit, and they are of course identical in the high-fluence limit. We have not looked into the magnitude of deviations from stoichiometric sputtering at intermediate fluences, but the magnitude of the difference between the resultant stationary profiles is substantial.

These features need to be taken into account in the interpretation of data on alloy and isotope sputtering. Quantities affected immediately are the distribution in energy and angle of ejected species as well as SIMS (secondary ion mass spectrometry), AES (Auger electron spectroscopy) and ISS (ion surface scattering) signals. The need to study these effects has been recognized many years ago, in particular after the recognition of the role of surface segregation. That point is accentuated even further in view of the apparent ability of moderate preferential sputtering alone to cause depletion of one species at the very surface.

A few examples illustrating the role of mixing and segregation have been included, firstly to demonstrate the scope of the formalism and, secondly, in order to not to let the results illustrating the isolated role of preferential sputtering stand alone. Indeed, feeding of preferential sputtering by mixing, diffusion, and segregation may be expected to lead to smoother concentration profiles [18,19], and this is to some extent corroborated by the present results. On the other hand, the condition of vanishing segregation currents at the surface defines the derivative of all $N_{i}(x)$ at $x=0$. Together with the stability condition and the requirement of stoichiometric sputtering. this imposes strong bounds on the near-surface composition profile in the presence of segregation currents. These features are wellknown in qualitative terms. The present formalism is hoped to shed more light on quantitative features.

\section{Appendix: Packing Condition and Balance Equa- tion for Variable Atomic Volume}

This appendix serves to demonstrate that the packing condition (1) is satisfied for all fluences, provided that it is satisfied at one particular instant, and that $N_{i}$ obeys the balance equation (2) with $h(x)$ given by eq. 10 .

Starting at

$$
\frac{\partial}{\partial \Phi} \sum_{i} \Omega_{i} \nu_{i}=\sum_{i} \frac{\partial \Omega_{i}}{\partial \Phi} N_{i}+\sum_{i} \Omega_{i} \frac{\partial N_{i}}{\partial \Phi}
$$


and inserting eq. (2) for $\partial N_{i} / \partial \Phi$ we find

$$
\frac{\partial}{\partial \Phi} \sum_{i} \Omega_{i} N_{i}=\sum_{i} \frac{\partial \Omega_{i}}{\partial \Phi} N_{i}+\sum_{i} \Omega_{i} L_{i} N_{i}-h(x)+\sum_{i} \frac{\partial \Omega_{i}}{\partial x} N_{i} \int_{0}^{x} d x^{\prime} h\left(x^{\prime}\right)
$$

or, by insertion of eq. (10) for $h(x)$,

$$
\frac{\partial}{\partial \Phi} \sum_{i} \Omega_{i} N_{i}=\sum_{i} N_{i}\left(\frac{\partial \Omega_{i}}{\partial \Phi}-\frac{\partial \Omega_{i}}{\partial N_{j}} L_{j} N_{j}+\frac{\partial \Omega_{i}}{\partial x} \int_{0}^{x} d x^{\prime} h\left(x^{\prime}\right)\right) .
$$

After insertion of functional derivatives for $\partial N_{i} / \partial \Phi$ and $\partial N_{i} / \partial x$, eq. (38) reduces to

$$
\frac{\partial}{\partial \Phi} \sum_{i} \Omega_{i} N_{i}=\sum_{i} N_{i} \frac{\partial \Omega_{i}}{\partial N_{j}}\left(\frac{\partial N_{j}}{\partial \Phi}-L_{j} N_{j}+\frac{\partial N_{j}}{\partial x} \int_{0}^{x} d x^{\prime} h\left(x^{\prime}\right)\right) .
$$

Finally, insertion of eq. (2) for $\partial N_{j} / \partial \Phi$ leads to

$$
\frac{\partial}{\partial \Phi} \sum_{i} \Omega_{i} N_{i}=-h(x) \sum_{i} N_{i} N_{j} \frac{\partial \Omega_{i}}{\partial N_{j}}
$$

which vanishes in view of eq. (11). This proofs the statement.

\section{Acknowledgements}

Thanks are due to N.Q.Lam for numerous stimulating discussions. This work was supported by a grant from the Danish Natural Science Research Council (SNF) as well as a visiting appointment to A.Oliva provided by the Faculty of Science, Odense University. The work at Argonne was supported by the U.S.Department of Energy, Office of Basic Energy Sciences, under Contract no. W-31-109-ENG-38.

\section{References}

[1] G.Betz and G.K.Wehner, in Sputtering by Particle Bombardment II, edited by R.Behrisch, Top. Appl. Phys. 52 (1983) 11

[2] T.Asada and K.Quasebarth, Z. Phys. Chem. A 143 (1929) 435

[3] N.Andersen and P.Sigmund, Mat. Fys. Medd. Dan. Vid. Selsk. 39 no. 3 (1974) 1

[4] E.Gillam, J. Phys. Chem. Solids 11 (1959) 55

[5] H.W.Pickering, J. Vac. Sci. Technol. 13 (1976) 618

[6] A.Turos, W.F.v.d.Weg, D.Sigurd, and J.W.Mayer, J. Appl. Ptys. 45 (1974) $2 \pi 77$

[7] T.Ishitani and R.Shimizu, Appl. Phys. 6 (1975) 241

[8] R.Kelly and J.B.Sanders, Nucl. Instrum. Methods 132 (1976) 335

[9] P.Sigmund, J. Appl. Phys. 50 (1979) 7261 
[10] A.Gras-Marti and P.Sigmund, Nucl. Instrum. Methods 180 (19S1) 211

[11] H.H.Brongersma, M.J.Spaarnay, and T.M.Buck, Surf. Sci. 71 (1978) 657

[12] H.H.Andersen, J. Vac. Sci. Technol. 16 (1979) 770

[13] T.Okutani, M.Shikata, and R.Shimizu, Surf. Sci. 99 (1980) L410

[14] R.Kelly, in Symposium on Sputtering, edited by P.Varga et al., Inst. Allg. Physik, Techn. Univ. Wien (1980) 390

[15] P.Sigmund, J. Vac. Sci. Technol. 17 (1980) 396

[16] P.Sigmund, in Sputtering by Particle Bombardment I, edited by R.Behrisch, Top. Appl. Phys. 47 (1981) 9

[17] P.Sigmund, A.Oliva, and G.Falcone, Nucl. Instrum. Methods 194 (1982) 541

[18] H.H.Andersen, in Physics of Ionized Gases 1980, edited by M.Matic, Boris Kidric Institute, Belgrade (1980) 421

[19] H.H.Andersen, in Advances in Ion Implantation, edited by J.M.Poate and J.S.Williams, Academic Press, New York (1984) 127

[20] W.O.Hofer and U.Littmark, Phys. Lett. 71A (1979) 457

[21] N.Q.Lam, Scann. Microsc. Suppl. 4 (1990) 311

[22] N.Q.Lam and P.Sigmund, Mat. Fys. Medd. Dan. Vid. Selsk., to be published

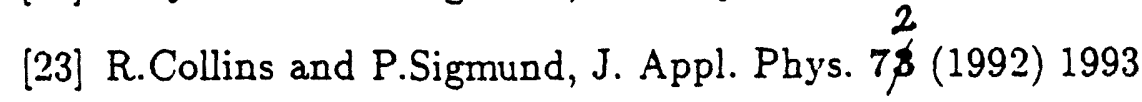

[24] A.D.Marwick, J. Phys. F 8 (1978) 1849

[25] N.Itoh and K.Morita, Radiat. Eff. 80 (1984) 163

[26] J. du Plessis, G.N. van Wyk, and E.Taglauer, Surf. Sci. 220 (1989) 381

[27] R.Collins, Nucl. Instrum. Methods B 2 (1984) 809

[28] A.Oliva, R.Kelly, and G.Falcone, Surf. Sci. 166 (1986) 403

[29] G.Falcone and A.Oliva, Appl. Phys. A 33 (1984) 175

[30] G.Falcone and P.Sigmund, Appl. Phys. 25 (1981) 307

[31] J.A.Peinador, I.Abril, J.J.Jiménez-Rodriguez, and A.Gras-Marti. Phys. Rev. B 44 (1991) 2061

[32] W.Möller and W.Eckstein, Nucl. Instrum. Methods B 2 (1984) 814 


\section{Figure Captions}

Figure 1. Stationary distribution of 1-atoms in binary medium, sputtering and relaxation only; sputter cross section $\sigma_{i}=A_{i} \exp \left(-x / a_{i}\right) ; A_{1}=2 ; A_{2}=1 ; a_{1}=a_{2}=1$; $c_{1}=c_{2}=0.5 ; \Omega_{1}=\Omega_{2}=1$. Solid line: exact solution; dashed line: weakly preferential; thin line: low-fluence profile, eq. (34) at fluence $\delta \Phi=\Phi^{\text {sat }}$, eq. (36).

Figure 2. Influence of the depth of origin of sputtered atoms on the stationary profile; sputtering only; $\sigma_{i}(x)=A_{i} \exp \left(-x / a_{i}\right) ; A_{1}=1.5 ; A_{2}=1$. Surface concentrations $N_{1}(x=0)$ have been marked by dots.

Curve a: $a_{1}=1 ; a_{2}=1.5 ;\left(Y_{1} / Y_{2}=1\right)$;

Curve b: $a_{1}=a_{2}=1 ;\left(Y_{1} / Y_{2}=1.5\right)$;

Curve c: $a_{1}=1.5 ; a_{2}=1 ;\left(Y_{1} / Y_{2}=2.25\right)$.

Figure 3. Influence of the amplitude of the sputter cross section on the stationary profile; sputtering only; $\sigma_{i}=A_{i} \exp \left(-x / a_{i} ; a_{1}=1.5 ; a_{2}=1\right.$.

Curve a: $A_{1}=1 ; A_{2}=1.5 ;\left(Y_{1} / Y_{2}=1\right)$;

Curve b: $A_{1}=A_{2}=1 ;\left(Y_{1} / Y_{2}=1.5\right)$;

Curve c: $A_{1}=1.5 ; A_{2}=1 ;\left(Y_{1} / Y_{2}=2.25\right)$.

Figure 4. Same as figure 2, but greater difference in sputter amplitudes; $A_{1}=2 ; A_{2}=1$.

Curve a: $a_{1}=1 ; a_{2}=1.5 ;\left(Y_{1} / Y_{2}=1.33\right)$;

Curve b: $a_{1}=a_{2}=1 ;\left(Y_{1} / Y_{2}=2\right)$;

Curve $c: a_{1}=1.5 ; a_{2}=1 ;\left(Y_{1} / Y_{2}=3\right)$.

Figure 5. Same as figure 3, but greater difference in sputter amplitudes; $a_{1}=1.5 ; a_{2}=1$.

Curve a: $A_{1}=1 ; A_{2}=2 ;\left(Y_{1} / Y_{2}=1.33\right)$;

Curve b: $A_{1}=A_{2}=1 ;\left(Y_{1} / Y_{2}=2\right)$;

Curve c: $A_{1}=2 ; A_{2}=1 ;\left(Y_{1} / Y_{2}=3\right)$.

Figure 6. Same as figure 3, but gretaer difference in sputter depth: $a_{1}=2 ; a_{2}=1$.

Curve a: $A_{1}=1 ; A_{2}=1.5 ;\left(Y_{1} / Y_{2}=1\right)$;

Curve b: $A_{1}=A_{2}=1 ;\left(Y_{1} / Y_{2}=1.5\right)$;

Curve c: $A_{1}=1.5 ; A_{2}=1 ;\left(Y_{1} / Y_{2}=2.25\right)$.

Figure 7 . Stationary profile due to mixing, sputtering, and relaxation; exponential relocation cross section, eq. (37) with parameters $B_{1}=1.3, B_{2}=0.7, b_{1}=0.8, b_{2}=$ $1.2, c_{1}=0.75, c_{2}=0.25, \Omega_{1}=\Omega_{2}=1$. Solid line: Iterative solution (present work); dashed line: numerical solution via time-dependent balance equation [29]; thin line: approximate analytical solution [17]; dot-dashed line: approximate solution given in ref. [28].

Figure 8. Influence of cascade mixing on concentration profile generated by preferential sputtering. Parameters as in figure 7 , but with the mixing current scaled by a factor $f$ increasing from 0 to 5 . 
Figure 9. Influence of surface segregation on sputter-generated profile; mixing ignored. Sputter parameters: $A_{1}=1.5 ; A_{2}=1.0 ; a_{1}=a_{2}=1.0$ (amplitude preferential) and $A_{1}=A_{2}=1 ; a_{1}=1.5 ; a_{2}=1.0$ (depth preferential). Segregation parameters: $V_{i}(x)=V_{0 i} \exp \left(-x / a_{i}^{\prime}\right) ; V_{01}=2 ; V_{02}=-2 ; a_{1}^{\prime}=a_{2}^{\prime}=1 ; D_{1}=D_{2}=0.02$; ion current $J=10$.

Figure 10. Same as figure 9 with sign of segregation reversed: $V_{01}=-2 ; V_{02}=2$. Depthpreferential sputtering only: $A_{1}=A_{2}=1 ; a_{1}=1.5 ; a_{2}=1.0$. Influence of ion current J.

Figure 11. Same as figure 10 for amplitude-preferential sputtering: $A_{1}=1.5 ; A_{2}=1.0 ; a_{1}=$ $a_{2}=1.0$. 
$-\frac{z}{x}$

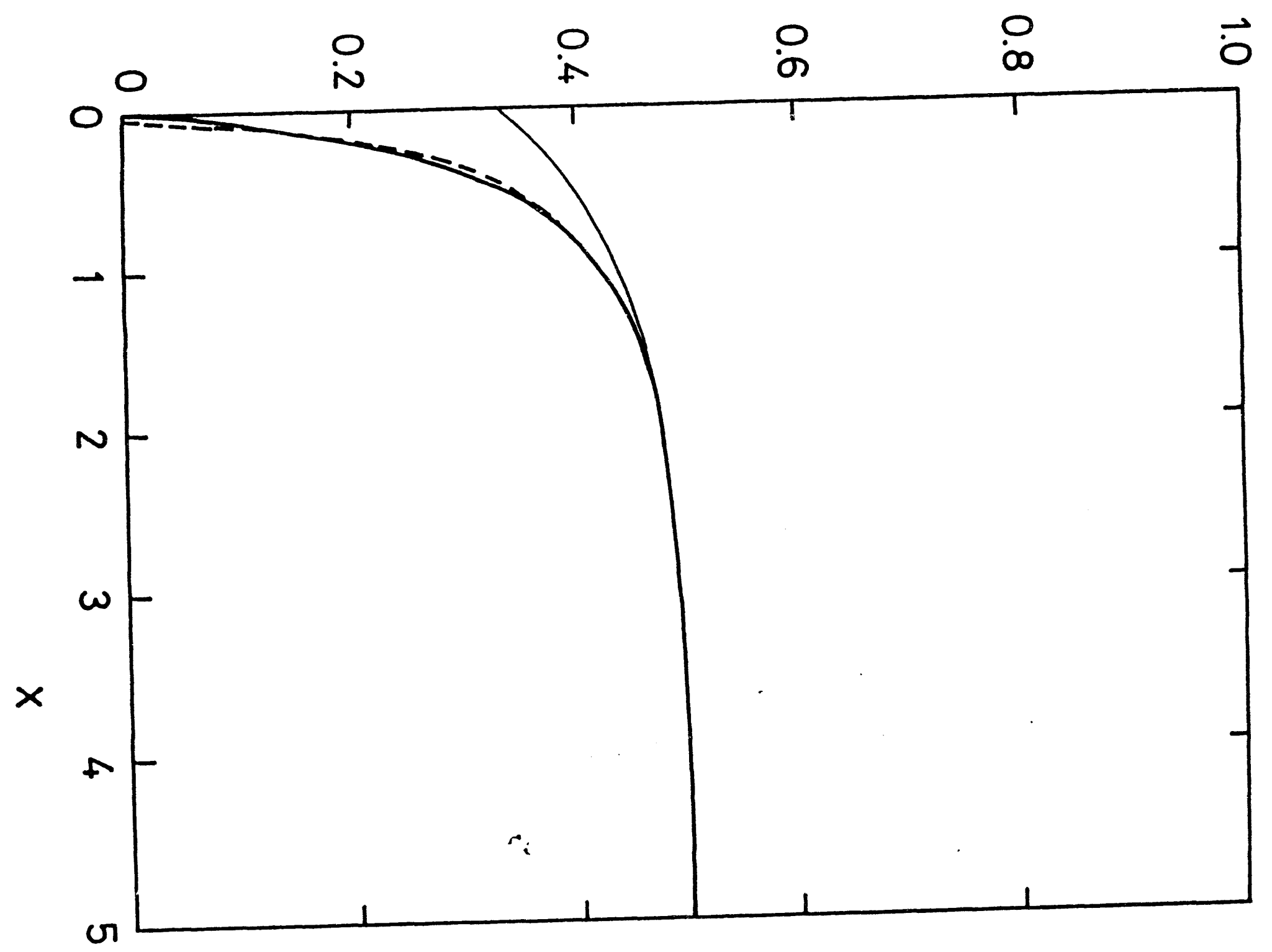




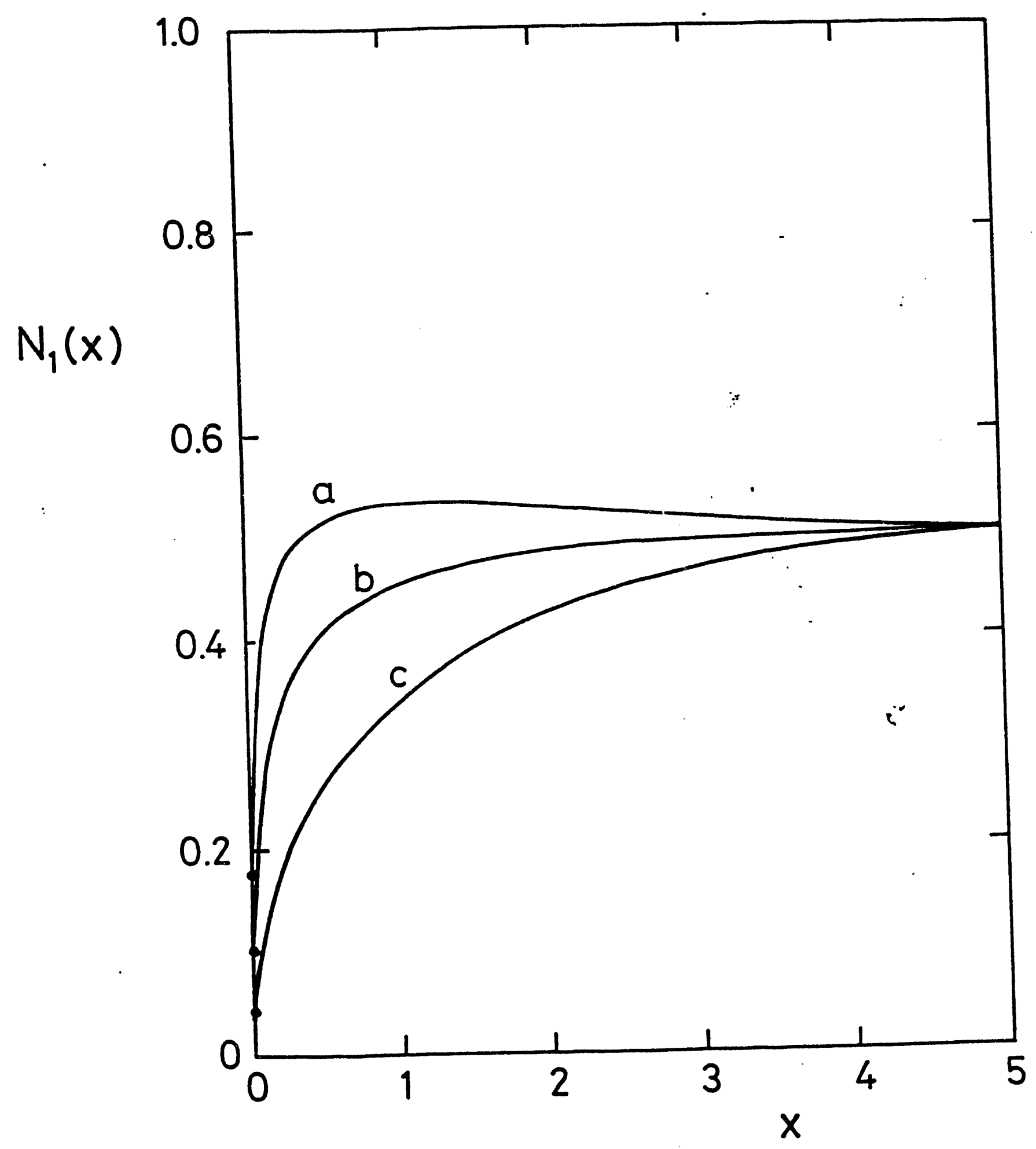


34

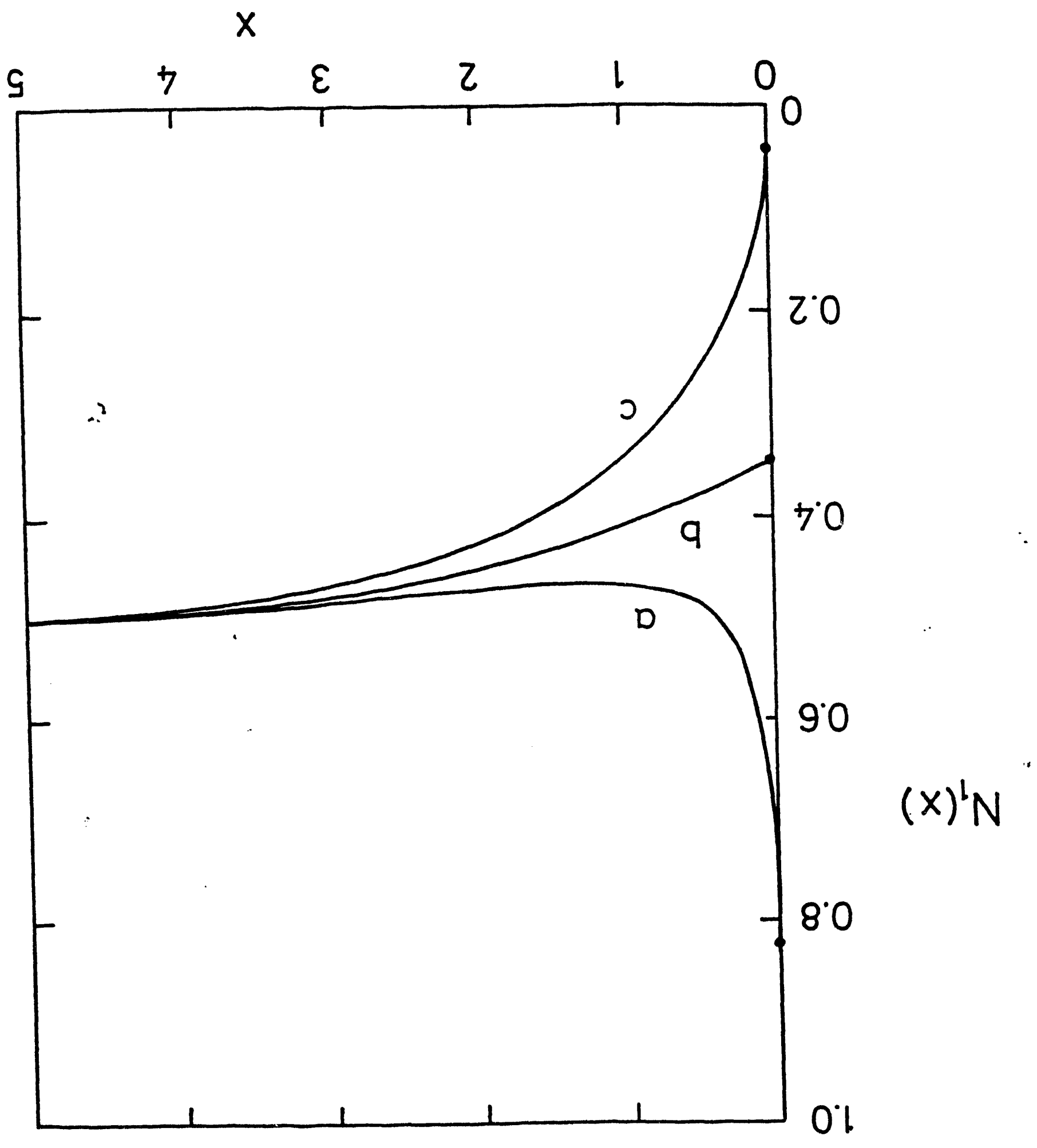




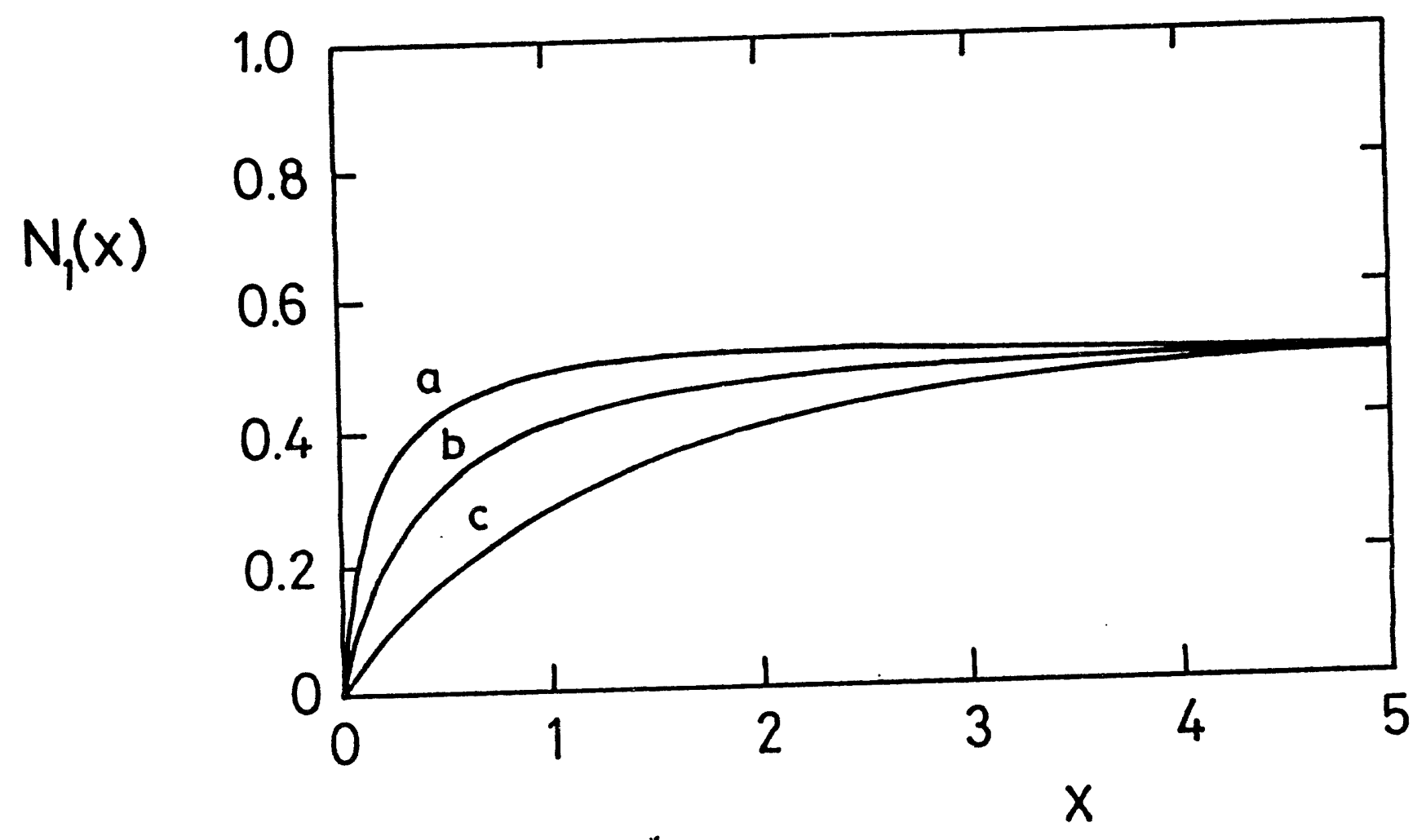




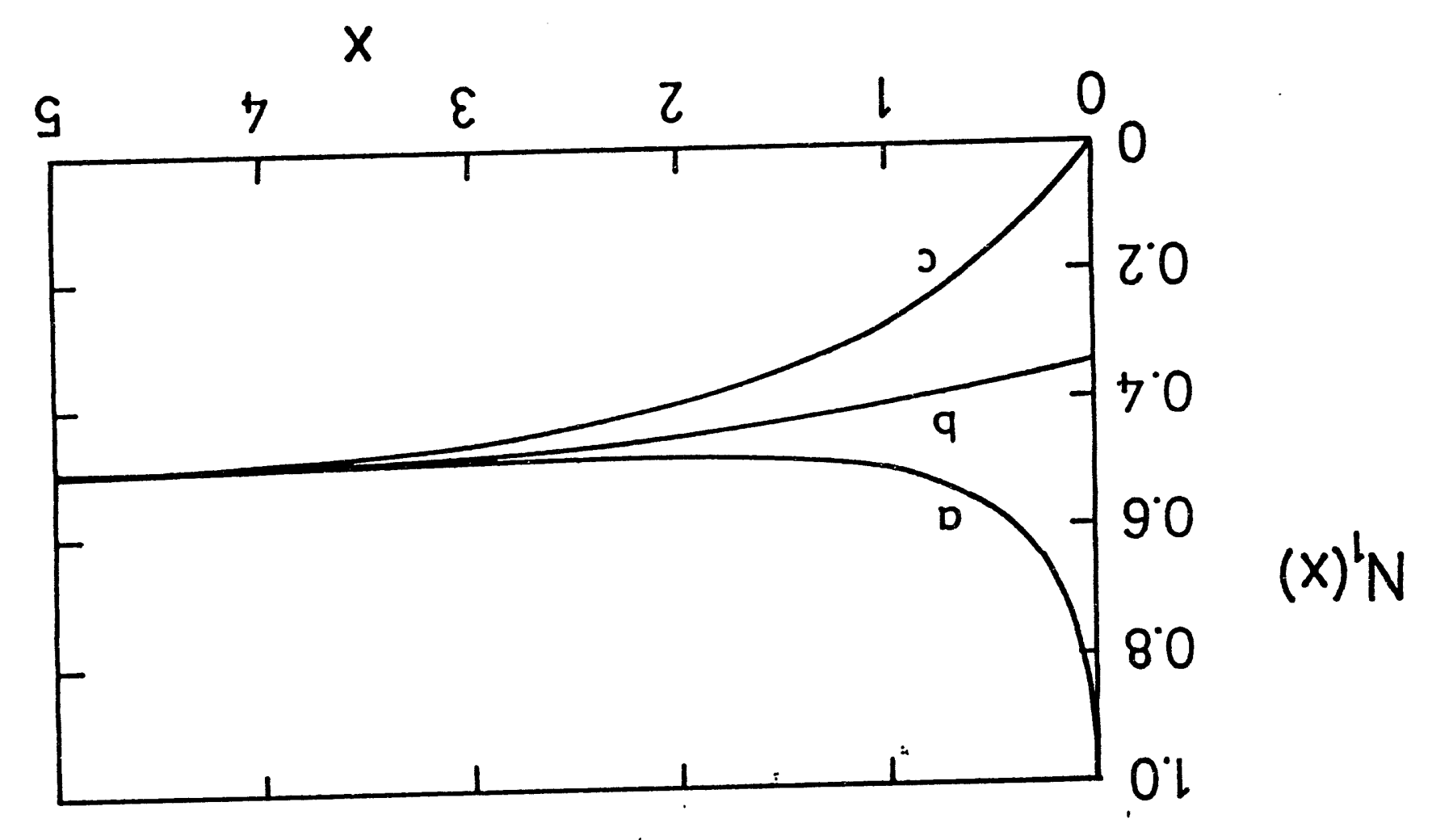

$r$ 


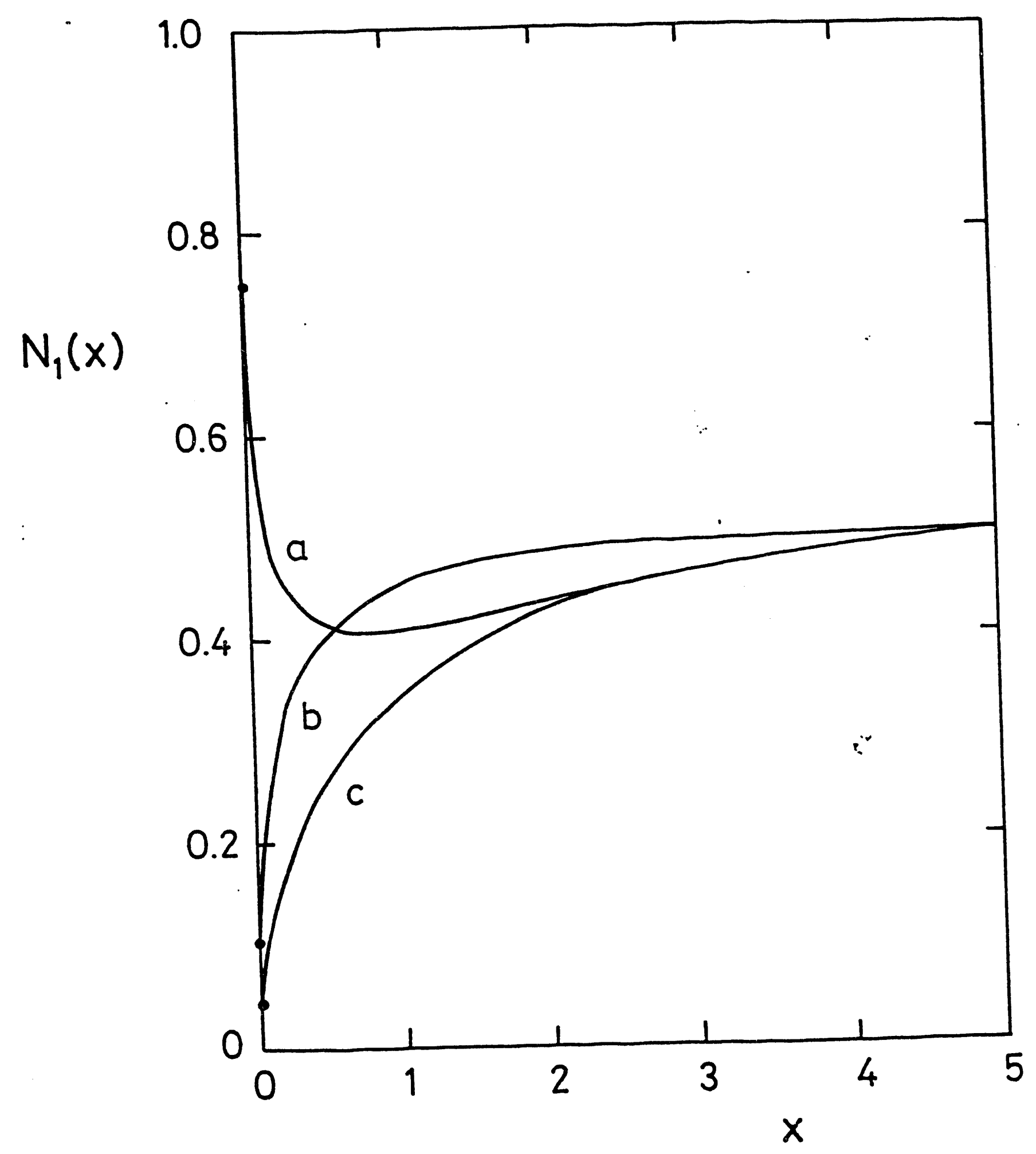

7. 


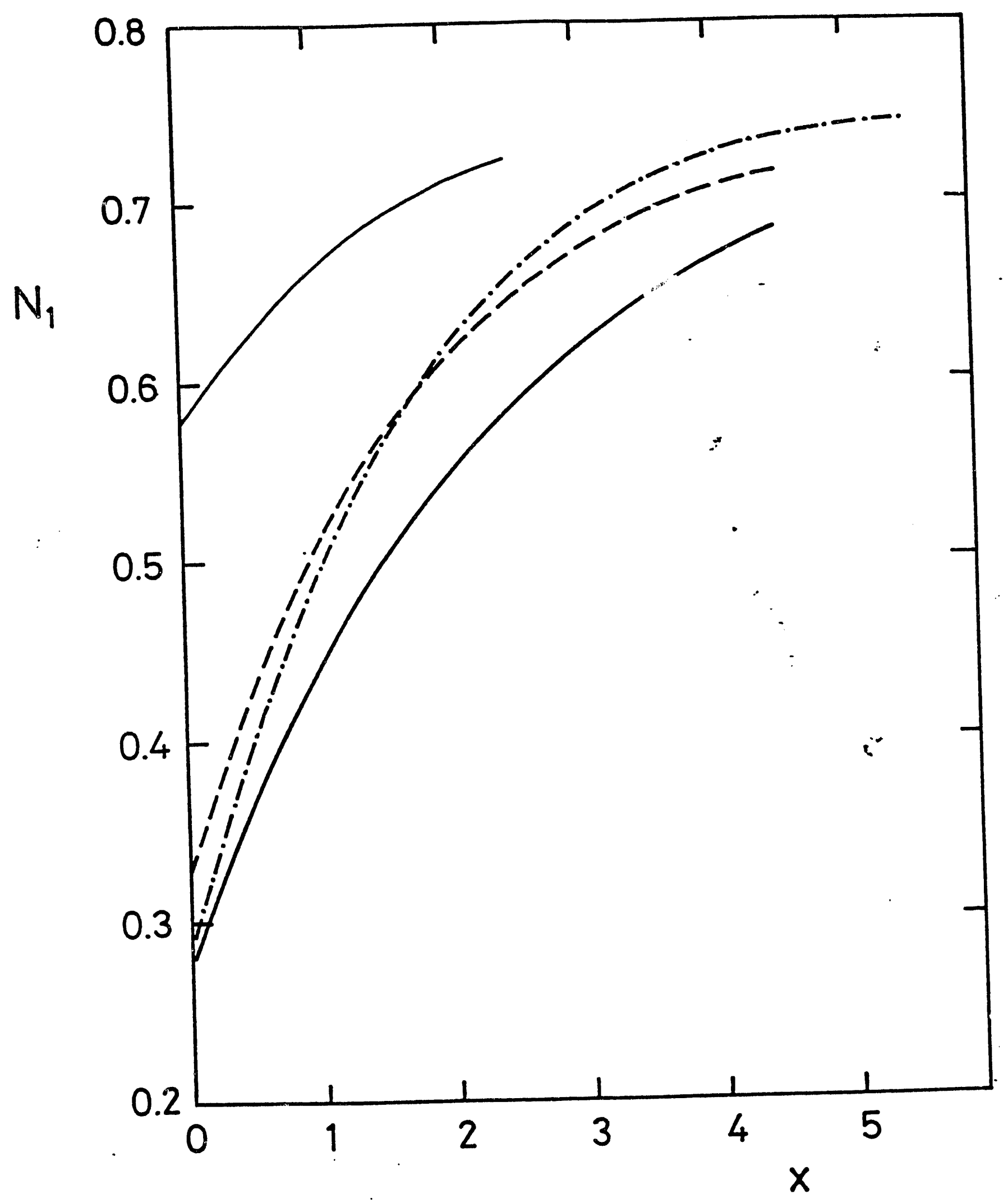

$F 7$ 


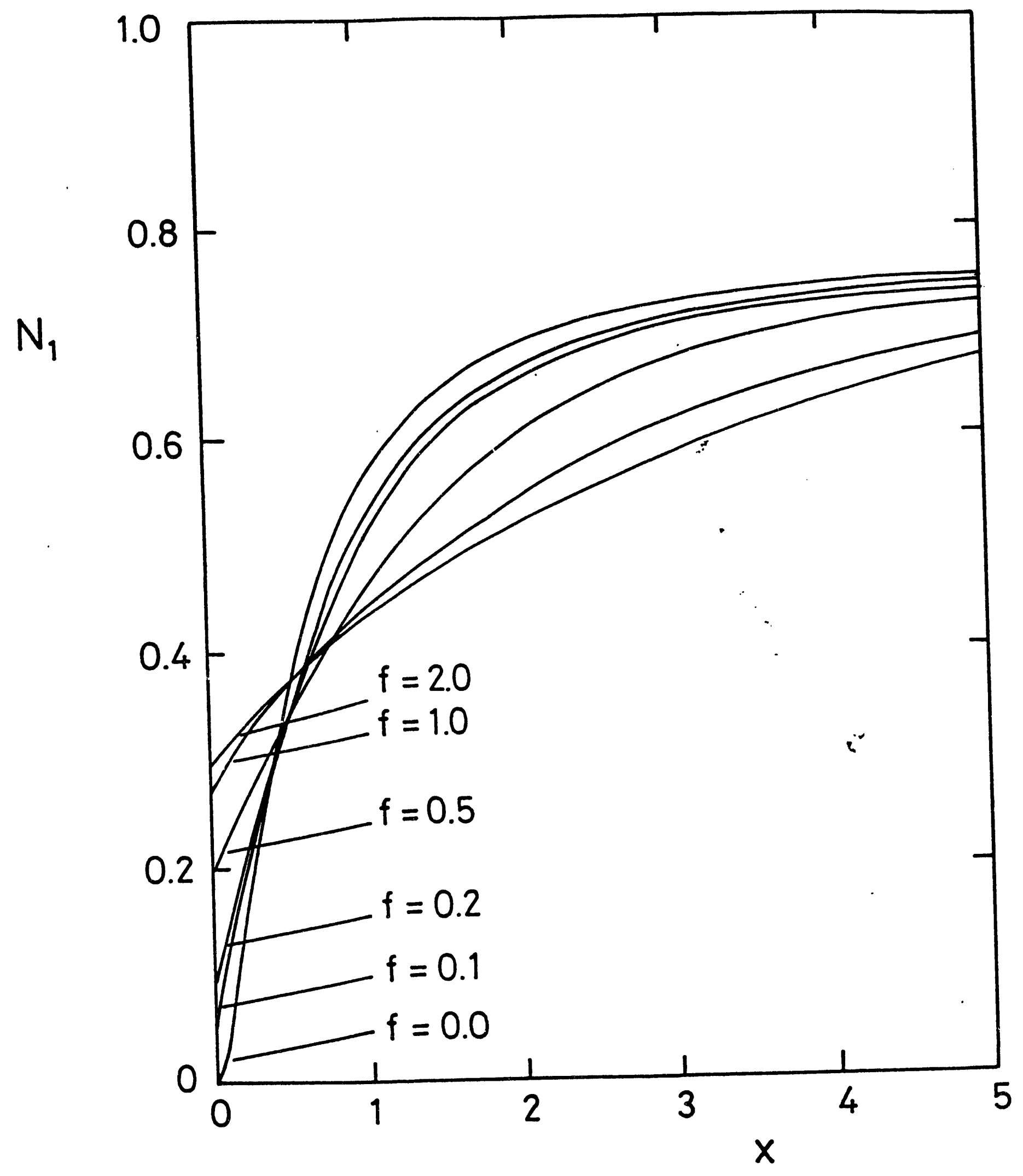

$=\quad r$ 
$=\ddots^{\prime}$

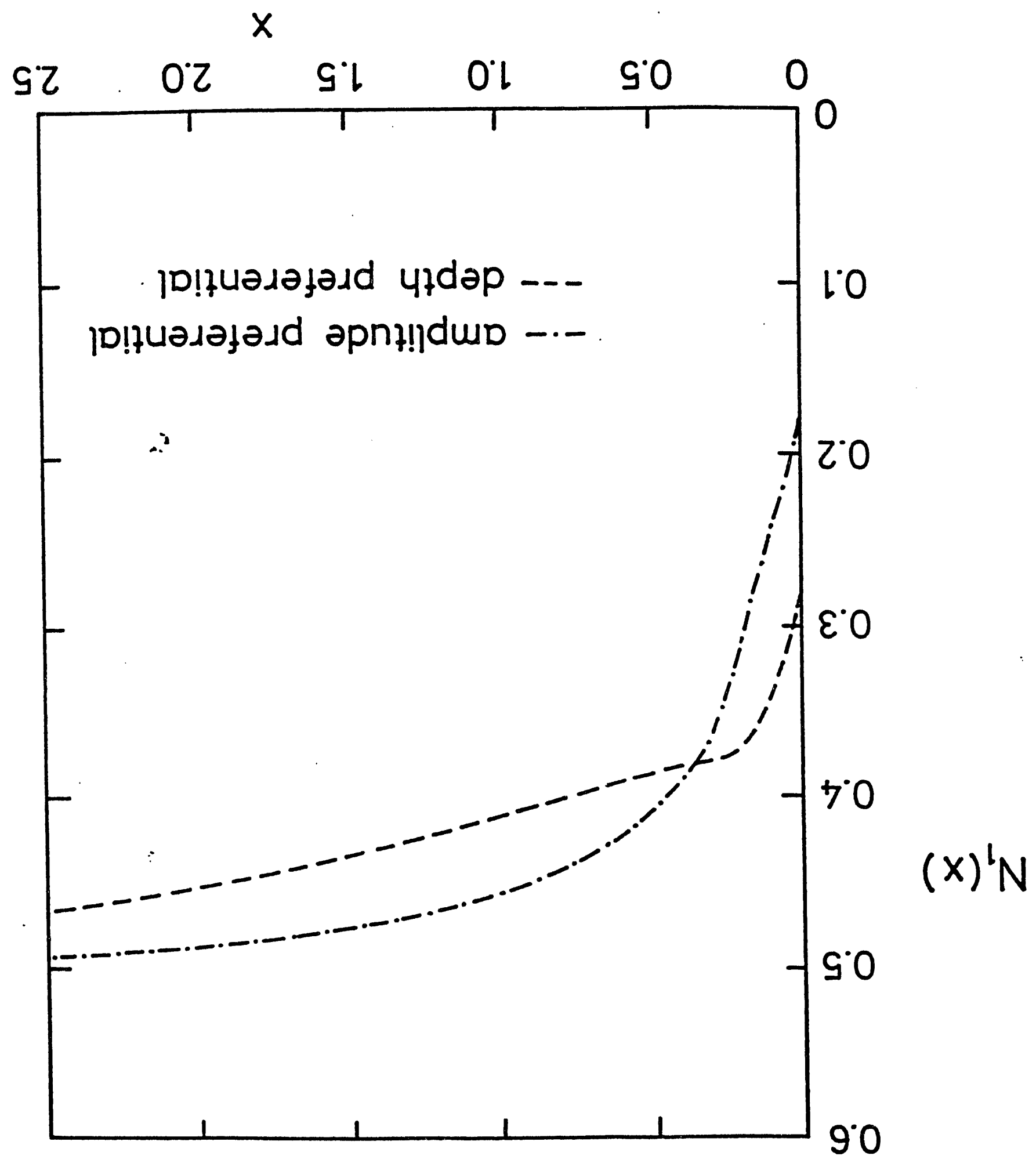




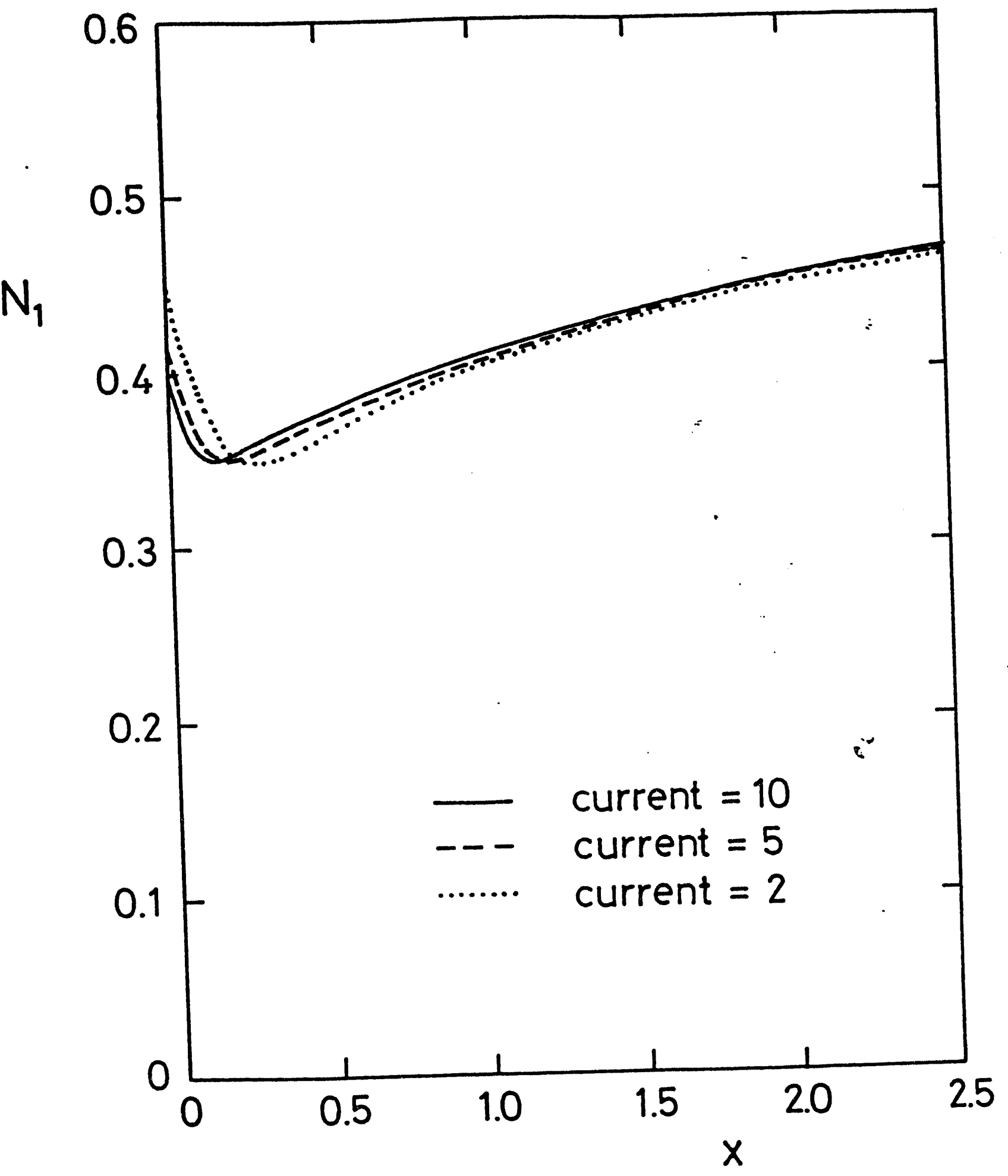




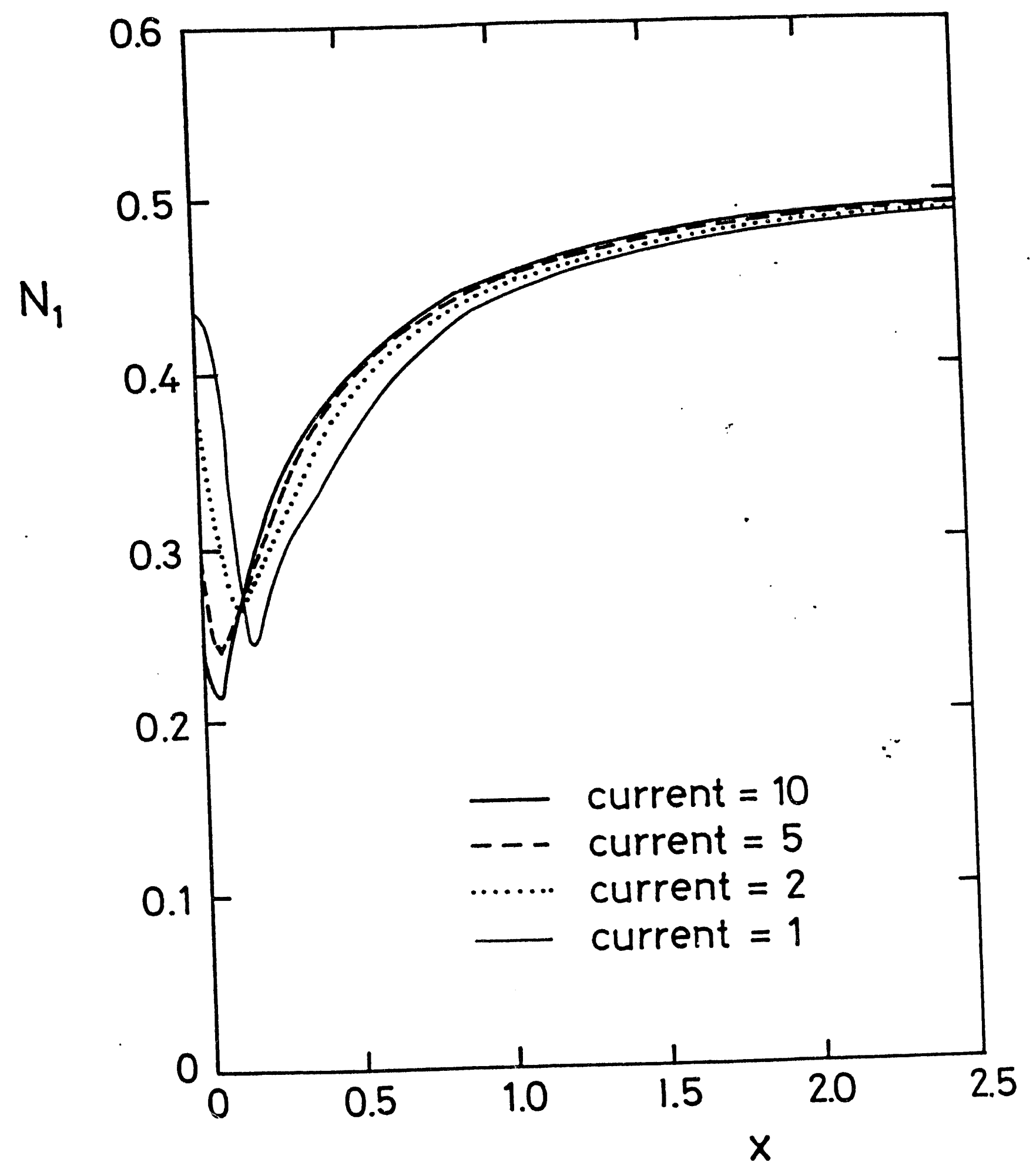



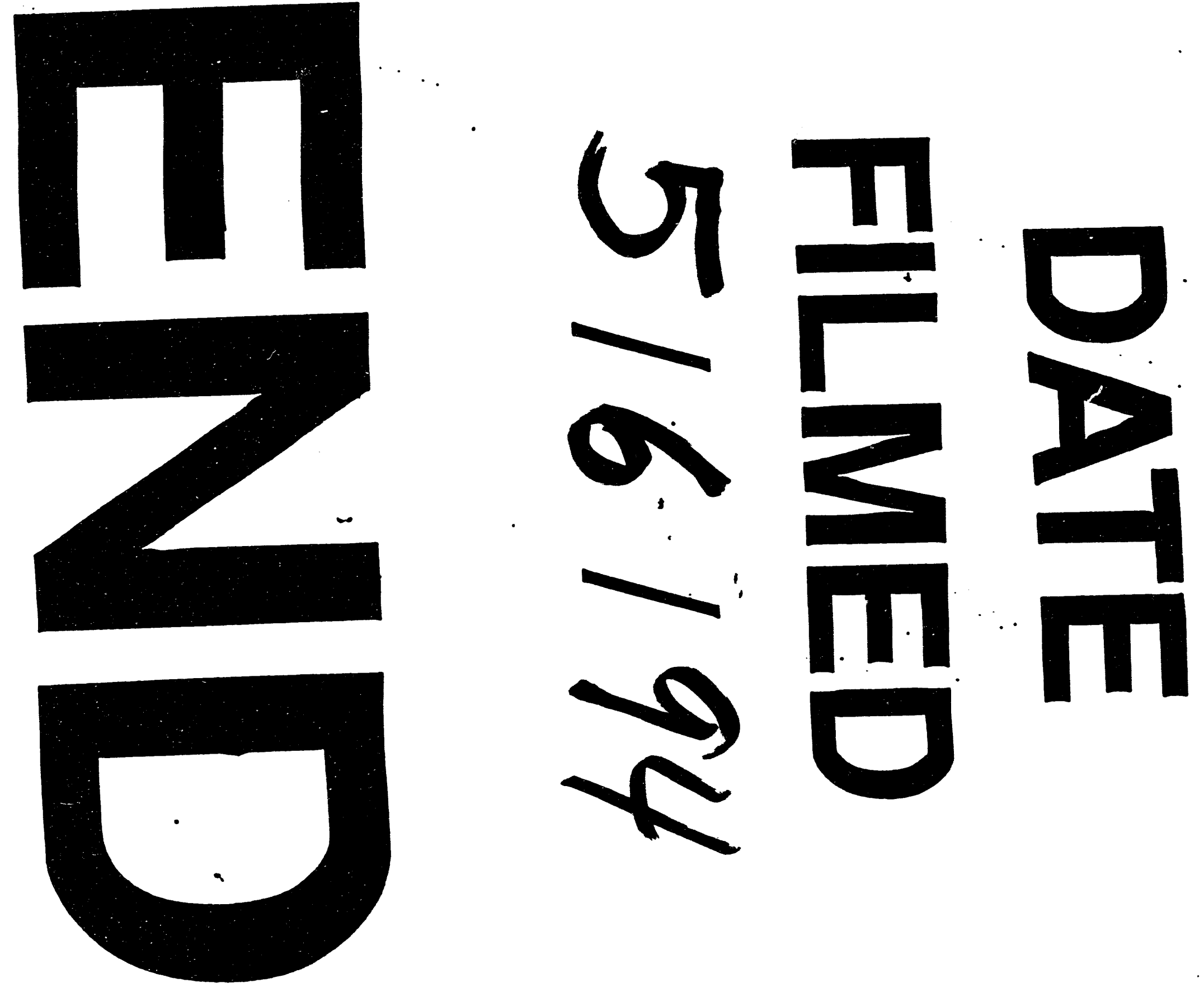
